Research Paper

\title{
Overexpression and potential roles of NRIP1 in psoriasis
}

\author{
Chao Luan ${ }^{1,2}$, Xu Chen ${ }^{1}$, Yu Hu${ }^{1}$, Zhimin Hao ${ }^{1}$, Jared M. Osland ${ }^{2}$, Xundi Chen ${ }^{2,3}$, \\ Skyler D. Gerber ${ }^{2,3}$, Min Chen ${ }^{1}$, Heng Gu ${ }^{1}$, Rong Yuan ${ }^{2}$ \\ ${ }^{1}$ Jiangsu Key Laboratory of Molecular Biology for Skin Diseases and STIs, Institute of Dermatology, Chinese Academy of \\ Medical Sciences \& Peking Union Medical College, Nanjing, Jiangsu, China \\ ${ }^{2}$ Department of Internal Medicine, Southern Illinois University School of Medicine, Springfield, IL, USA \\ ${ }^{3}$ Department of Medical Microbiology, Immunology and Cell Biology, Southern Illinois University School of Medicine, \\ Springfield, IL, USA \\ Correspondence to: Min Chen, email: drchenmin@126.com \\ Heng Gu, email: guheng@aliyun.com \\ Rong Yuan, email: ryuan@siumed.edu
}

Keywords: NRIP1, psoriasis, skin, T cells, animal model

Received: July 26, $2016 \quad$ Accepted: September 22, 2016

Published: September 30, 2016

\section{ABSTRACT}

Nuclear receptor interacting protein 1 (NRIP1, also known as RIP140) is a coregulator for various transcriptional factors and nuclear receptors, and has been shown to take part in many biological and pathological processes, such as regulating mammary gland development and inflammatory response.

The aim of this study is to investigate the expression of NRIP1 and to explore its roles in the pathogenesis of psoriasis. Thirty active psoriasis patients and $\mathbf{1 6}$ healthy volunteers were enrolled for this study. QRT-PCR analyses found that both NRIP1 and RelA/p65 were elevated in psoriatic lesions compared to psoriatic non-lesions and normal controls, and also overexpressed in peripheral blood mononuclear cell (PBMCs) of psoriasis patients. Suppression of NRIP1 in HaCaT cells could significantly inhibit cell growth and induce apoptosis, and the suppression of NRIP1 in CD4 ${ }^{+} \mathrm{T}$ cells isolated from psoriasis patients could downregulate the expression of RelA/p65 and decrease the secretion of IL-17. Furthermore, in Nrip1 knockout mice, IMQ-induced inflammation of skin was delayed and the RelA/p65 expression in lesions was reduced. In conclusion, our data suggests that NRIP1 is overexpressed both in skin and PBMCs of psoriasis patients and may be involved in the abnormal proliferation and apoptosis of keratinocytes, as well as the immune reaction through the regulation of RelA/p65. Therefore, NRIP1 may be a potential therapeutic target for psoriasis.

\section{INTRODUCTION}

NRIP1, one of the first isolated nuclear receptor transcriptional cofactors, works as an unconventional coregulator for many nuclear receptors. It is highly expressed in adipose tissue [1], liver [2] and skeletal muscle and mainly acts as a transcriptional co-repressor of genes involved in glucose uptake, glycolysis, fatty acid oxidation, mitochondrial biogenesis and oxidative phosphorylation to ultimately repress energy utilization [3]. However, accumulating studies have provided evidence that NRIP1 may also work as a co-activator for a variety of other transcription factors in numerous cellular responses, such as inflammatory gene expression in macrophages [4], amphiregulin expression in the ovary [5] and triglyceride synthesis in the liver [2]. In macrophages, NRIP1 functions as a co-activator for NF- $\mathrm{kB}$ through direct interaction with RelA/p65 and the transcriptional co-activator CREBbinding protein (CBP), and up-regulates the downstream inflammatory gene expression such as TNF- $\alpha$ and interleukin-6 [4]. In addition, a study also showed that NRIP1 expression was controlled by the E2 promotor binding factor (E2F) pathway, which may play a crucial role in gene transcription, cellular proliferation and apoptosis [6]. We recently reported that NRIP1 was overexpressed in human breast cancer and the suppression of NRIP1 could inhibit growth and induce apoptosis of breast cancer cells [7].

Psoriasis is a chronic inflammatory skin disorder affecting approximately $1-3 \%$ of the general population. It is characterized by keratinocytes hyper-proliferation and inflammatory cellular infiltrate in both dermis and epidermis. NF- $\mathrm{KB}$ is identified as a key mediator in the 
pathogenesis of psoriasis because of its involvement in inflammatory pathways, proliferation and apoptosis of various cell types [8]. Previous studies have elucidated that NF- $\kappa \mathrm{B}$ levels are elevated in both psoriatic plaques (PP), and uninvolved, clinically asymptomatic skin of psoriatic patients (PN) compared to normal healthy skin (NN). Numerous chemokines and cytokines in the pathogenesis of psoriasis are dependent on the NF$\kappa \mathrm{B}$ signaling pathway [9]. NF- $\kappa \mathrm{B}$ functions as a link between abnormal keratinocytes and immune cell states [10]. Various treatments that improve the symptoms of psoriasis, including epigallocatechin-3-gallate (EGCG), IL-12/23 and TNF- $\alpha$ inhibitors, could also suppress the expression or activity of $\mathrm{NF}-\kappa \mathrm{B}[9]$.

In order to evaluate the function of NRIP1 in cell growth, apoptosis and inflammation involved in psoriasis, we used human skin biopsies and peripheral blood mononuclear cells (PBMCs), HaCaT cells and $\mathrm{CD}^{+} \mathrm{T}$ cells isolated from psoriasis patients, along with an in vivo study of IMQinduced psoriasis in Nripl knockout mice. Our results

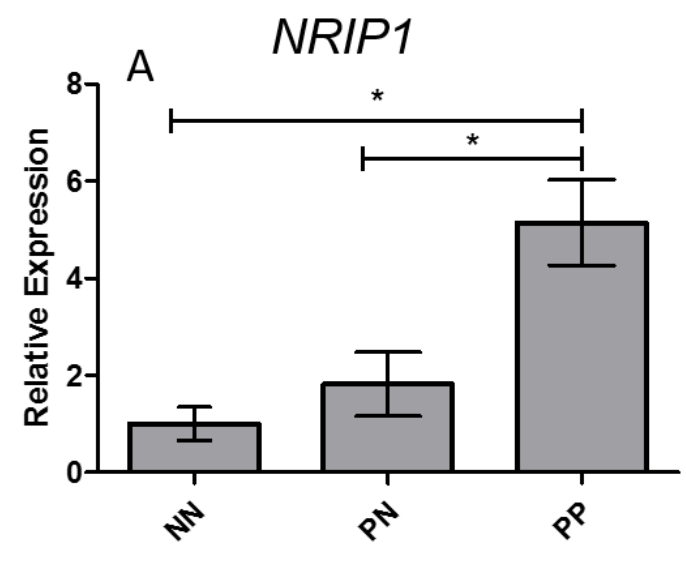

C

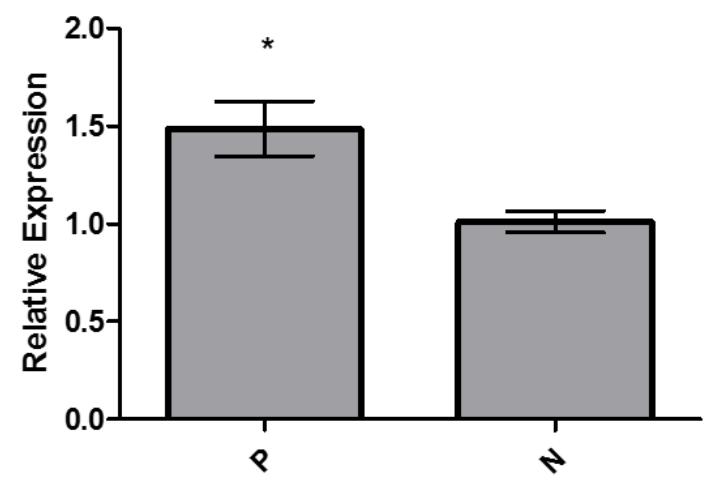

suggest that NRIP1 may play a role in the pathogenesis of psoriasis and may be a novel therapeutic target for psoriasis.

\section{RESULTS}

\section{Overexpression of NRIP1 and p65 in lesions and PBMCs of psoriasis patients}

In order to investigate its potential role in psoriasis, we first measured the expression of NRIP1 in the skin lesions and PBMCs of psoriasis patients. QRTPCR assays showed that mRNA of NRIP1 significantly increased in PP compared to PN (5.1430 \pm 0.8793 vs. $1.8170 \pm 0.6592, \mathrm{P}=0.0218)$, but no significant difference was observed between PN and NN (1.817 \pm 0.6592 vs. $1.000 \pm 0.3413, \mathrm{P}=0.4880$ ) (Figure $1 \mathrm{~A})$. The same trend was found in the p65 expression, which is significantly increased in PP compared to PN (5.4670 \pm 0.8819 vs. $1.4870 \pm 0.3091, \mathrm{P}=0.0006)$; but no significant difference was found between in PN and in

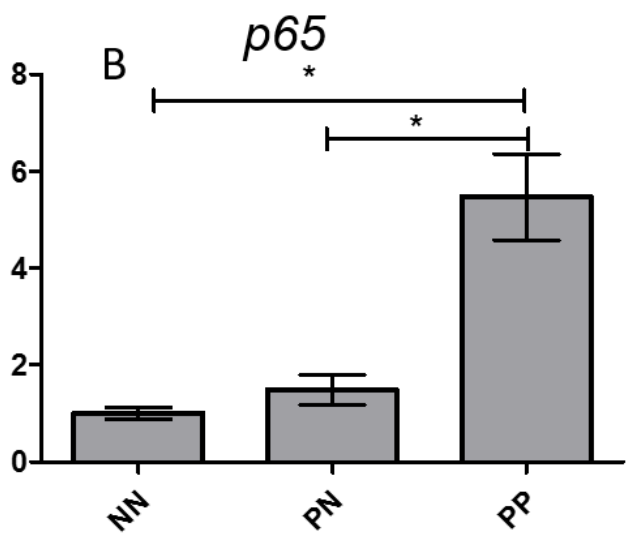

D

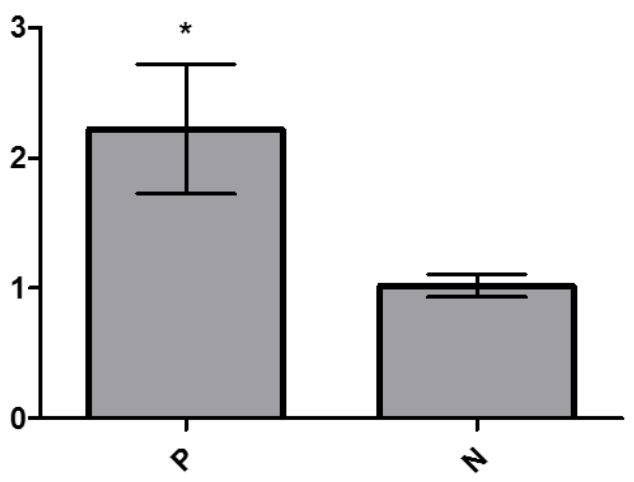

$$
\text { *: } P<0.05
$$

Figure 1: Expression of NRIP1 and p65 was elevated in lesions and PBMCs of psoriasis patients. Expression of NRIP1 mRNA A. and p65 mRNA B. was significantly increased in PP compared to PN and NN, respectively ( $<<0.05)$. In PBMCs, the expression of NRIP1 mRNA C. and p65 mRNA D. was remarkably elevated in psoriasis patients $(\mathrm{p}<0.05)$. PP: psoriatic lesions, PN: psoriatic nonlesions, NN: healthy controls, P: psoriasis patients, N: Normal persons 
$\mathrm{NN}(1.4870 \pm 0.3091$ vs. $1.000 \pm 0.1241, \mathrm{P}=0.4023)$ (Figure 1B). There was no significant correlation between the mRNA expression of NRIP1 and p65 in psoriatic lesions $(\mathrm{r}=-0.013, \mathrm{P}=0.974)$.

The previous study by our team also applied immunohistochemistry (IHC) on three types of skin biopsies, and found that expression of NRIP1 was significantly increased in both the epidermis and dermis of PP. NRIP1 positive cells were widely distributed in epidermis (keratinocytes and melanocytes) and dermis (lymphocytes, fibroblasts and epithelia cells), but not in the stratum corneum. There was no significant difference between PN and NN groups either in epidermis or dermis(data unpublished).

Expression of NRIP1 and p65 were also significantly elevated in PBMCs of psoriasis patients compared to healthy controls $(1.4860 \pm 0.1408$ vs. $1.0110 \pm 0.05578$, $\mathrm{P}=0.0165 \& 2.2220 \pm 0.4975$ vs. $1.019 \pm 0.08736, \mathrm{P}=$ 0.0385 , respectively. Figure $1 \mathrm{C} \& 1 \mathrm{D})$.

\section{Suppression of NRIP1 inhibited growth and induced apoptosis of $\mathrm{HaCaT}$ cells}

Excessive proliferation of keratinocytes is an important pathological feature of psoriasis, and most of the treatments that improve the condition of psoriasis could suppress the proliferation of the keratinocytes. In order to illustrate the in vitro significance of NRIP1 on the proliferation of keratinocytes, we applied shRNA targeting NRIP1 (shNRIP1) to suppress NRIP1 expression in $\mathrm{HaCaT}$ cells, and used non sense shRNA ( $\mathrm{shCON}$ ). The qRT-PCR results (Figure 2A) showed a 70\% depletion of NRIP1 expression in $\mathrm{HaCaT}$ cells (mRNA expression of shNRIP1 $1.0 \pm 0.66$, shCON $0.30 \pm 0.07$ ). These HaCat cells, transfected with shNRIP1 and shCON, were used for the next experiments.

As the 3-(4,5-dimethylthiazol-2-yl)-2,5diphnyltetrazolium bromide (MTT) assay showed (Figure 2B), shNRIP1 reduced the growth of HaCaT cells significantly at the $48 \mathrm{~h}, 72 \mathrm{~h}, 96 \mathrm{~h}$ and $120 \mathrm{~h}$ time points after being seeded $(\mathrm{P}<0.05)$.

In order to illustrate the reasons for the reduction of cell growth by suppressing NRIP1, we detected cell proliferation and apoptosis of $\mathrm{HaCaT}$ cells by PI staining assay and Annexin- $\mathrm{V}$ assay and flow cytometry. As shown in Figure 2C\&2D, suppressing NRIP1 remarkably induced apoptosis of HaCat cells $(\mathrm{P}<0.05)$; however, there is no clear trend of alteration in $\mathrm{G} 0 / \mathrm{G} 1, \mathrm{~S}$ and $\mathrm{G} 2 / \mathrm{M}$ phases between shCON and shNRIP1 treatment of HaCaT cells. These results suggest that suppression of NRIP1 could induce apoptosis of $\mathrm{HaCaT}$ cells.

As the above data showed that both NRIP1 and p65 were overexpressed in psoriasis lesions, we applied western blot assay to detect the function of silencing NRIP1 on the expression of p65 in HaCaT cells. The western blot data (Figure 2E) showed that the expression of p65 was reduced significantly by the suppression of NRIP1 in $\mathrm{HaCaT}$ cells.

\section{Suppression of NRIP1 reduced expression of p65 and decreased secretion of IL-17 in CD4+T cells}

CD4+ $\mathrm{T}$ cells are the major resource of the inflammatory cytokines, such as IL-17. To investigate the function of NRIP1 in regulating the secretary profile of CD4+ T cells, we isolated CD4+ T cells from psoriasis patients and used siRNA to silence NRIP1 (siNRIP1) expression. As examined by qRT-PCR at $48 \mathrm{~h}$ and $72 \mathrm{~h}$ after siRNA treatment (Figure 3A), the most significant NRIP1 mRNA inhibition of siNRIP1 treatment compared to siCON treatment $(\mathrm{P}<0.05)$ was detected at $72 \mathrm{~h}$. We then measured the expression of p65 mRNA at the same time points and found the suppression of NRIP1 downregulated the expression of $p 65 \mathrm{mRNA}$ at each time point. The most significant reduction occurred at $72 \mathrm{~h}$, which is in accordance with the expression of NRIP1 mRNA (Figure 3B). The supernatants of culture medium were also collected for ELISA. The result showed that the concentration of IL-17 was significantly decreased at $72 \mathrm{~h}(\mathrm{p}<0.05$, Figure 3C).

\section{The depletion of Nrip1 delays IMQ-induced skin inflammation in vivo}

Overexpression of NRIP1 in the tissues of patients and the effects of silencing NRIP1 in HaCaT cells strongly suggested that the depletion of NRIP1 may improve the skin lesion of psoriasis. To test this hypothesis, we compared the psoriasis-like reactions induced by IMQ treatment between wild-type (WT) B6 and Nrip 1-/- mice. Mice were treated with 5\% IMQ cream on their shaved backs for five consecutive days. Two or three days after application, signs of erythema, scaling, and thickness began to occur. On the third day, indicated by erythema and scaling, the skin inflammation of Nrip 1-/- mice was weaker than WT mice. By the fifth day, both groups of mice developed similar lesions resembling psoriasis (Figure 4A). The independent scores and cumulative scores are depicted in Figure 4B. Compared with WT mice, the scores of erythema and scaling of Nrip1-/- mice were lower on the third day of treatment. However, on the fourth day, scores of erythema and scaling of both groups are approaching to the same level. Cumulative scores showed the same trend. There was no difference in the thickness scores between the two groups. Then we further measured the thickness of epidermis, using H\&E stained skin sections from WT and Nrip1-/- mice, both in IMQ treated and untreated areas. The H\&E stained sections were digitally imaged, then the thickness of epidermis was measured in 10 microscopic fields (40x) for each core in blind studies 

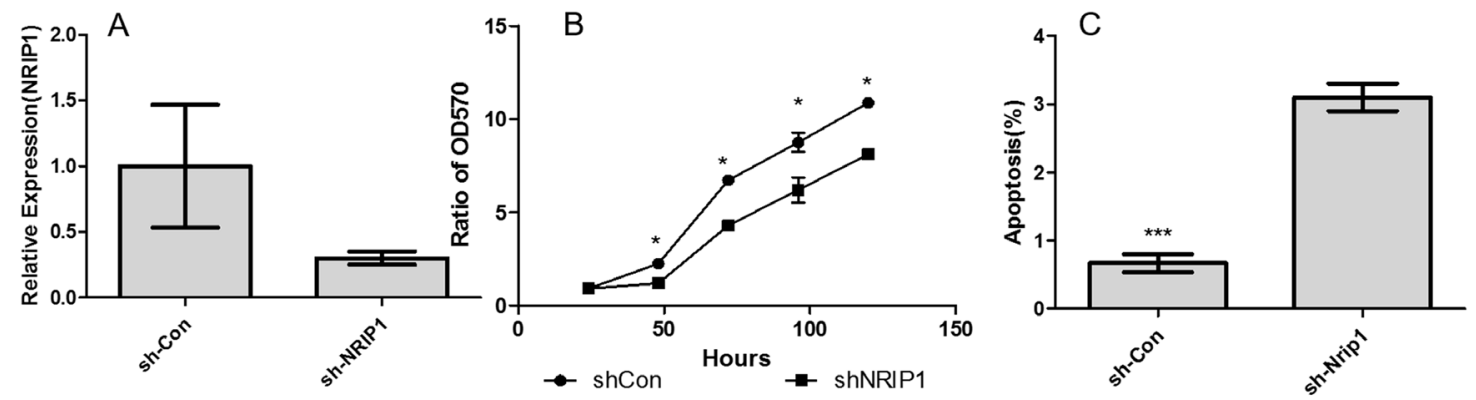

$*: \mathrm{P}<0.05$

$* * *: P<0.001$

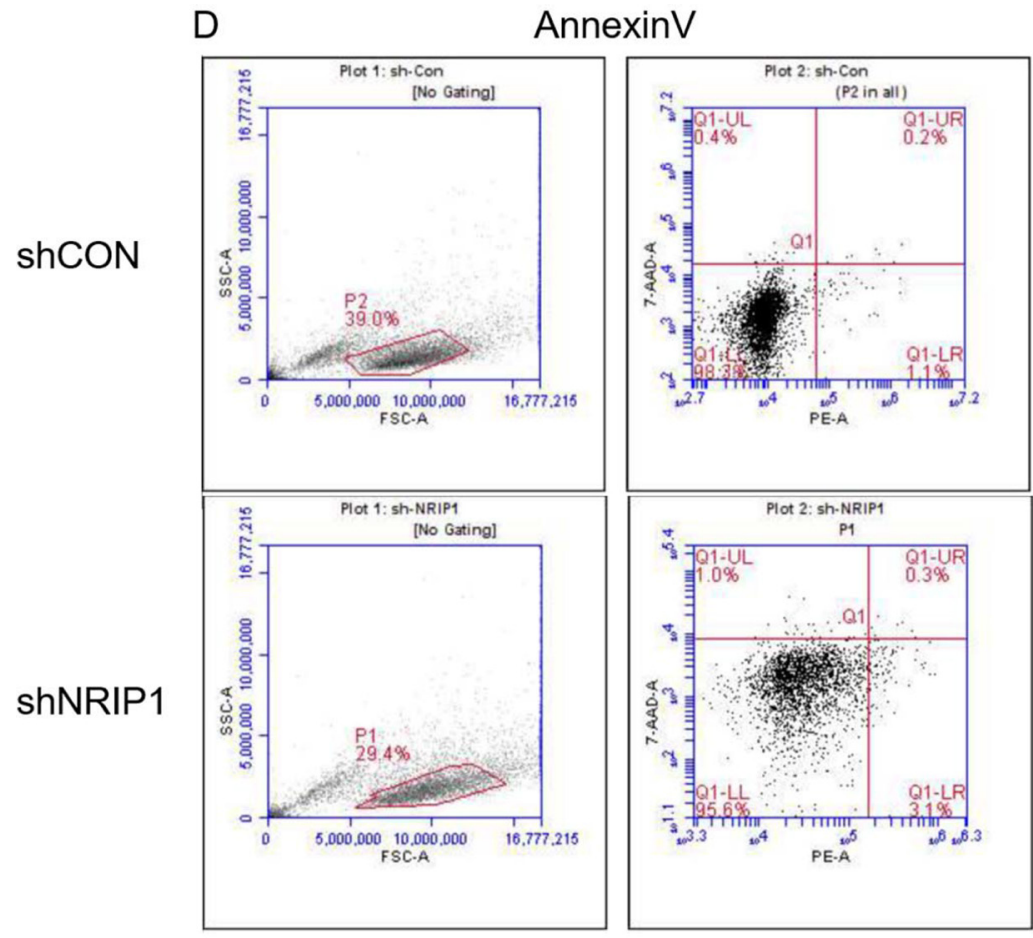

E shCON sh-NRIP1

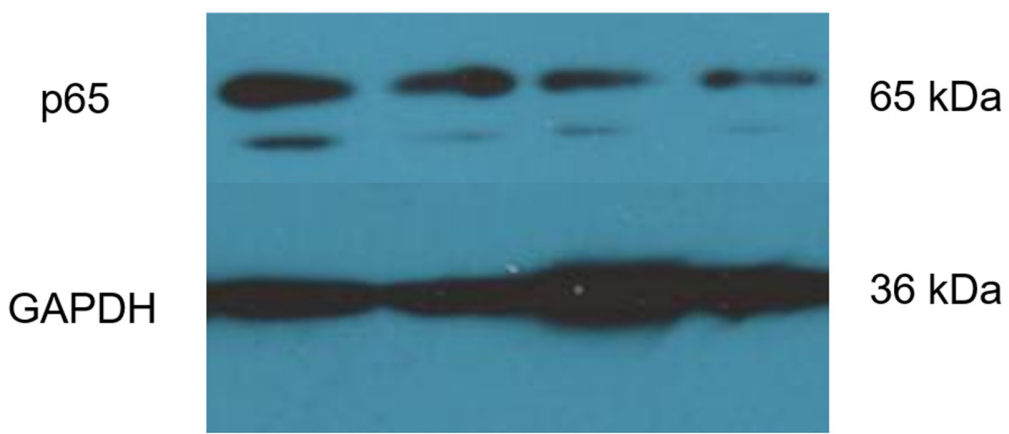

Figure 2: Suppression of NRIP1 inhibited the growth and induced apoptosis of HaCaT cells. A. shNRIP1 suppressed the NRIP1 expression in HaCaT cells. B. MTT assay showed shNRIP1 reduced the growth of HaCaT cells significantly at the 48h, 72h, 96h and $120 \mathrm{~h}$ time points $(\mathrm{p}<0.05)$ after being seeded, respectively. C\&D. Annexin V showed that shNRIP1 induced the apoptosis of HaCaT cells significantly $(\mathrm{p}<0.001)$. E. Western blot result showed that the expression of $\mathrm{p} 65$ was remarkably reduced by shNRIP1 transfection in $\mathrm{HaCaT}$ cells. 
conducted by two researchers, one of which is a clinical dermatologist. As the results showed in Figure 4C, after 5 days of IMQ treatment, thickness of epidermis in treated area was increased in both two groups, but the increment was significantly reduced in $\mathrm{Nrip}^{-/}$mice, compared to WT mice.

\section{The depletion of Nrip1 suppresses the IMQ- induced expression of $\mathbf{p 6 5}$}

Analysis of H\&E-stained sections of IMQ-treated skin from these two different genotypes of mice showed typical characteristics of psoriasis biopsy (Figure 5A), such as hyper-proliferative keratinocytes, parakeratosis and the absence of a granular layer. However, the inflammatory cell infiltration in the dermis of Nrip 1-/- mice was much less than that in WT mice, which suggested that the depletion of Nripl may reduce inflammation caused by IMQ.

In order to detect the function of Nrip1 deficiency on NF- $\kappa \mathrm{B}$ during IMQ treatment, IHC staining was used to detect the expression of p65 in skin sections from WT and Nrip1-/- mice, both in IMQ treated and untreated area. The p65 stained sections were digitally imaged. The IHC results (Figure 5B) showed that compared to WT mice, p65 positive cells within IMQ treated skin were decreased in epidermis and dermis of $\mathrm{Nrip}^{-/ /}$mice, respectively.

\section{DISCUSSION}

NRIP1 interacts as a co-activator or co-repressor with numerous nuclear receptors, including peroxisome proliferatoractivated receptors (PPARs), liver X receptor (LXR), estrogen receptor-related receptor (ERR), and estrogen receptor (ER) [2]. A recent study revealed that NRIP1 may stimulate the activity of NF- $\mathrm{B}$ by forming a trimeric complex with RelA and CBP [4], and upregulate downstream inflammatory genes, including TNF- $\alpha$ and
IL-6, both of which play crucial roles in the pathogenesis of psoriasis. Other studies have shown that except for macrophages, NRIP1 may also work as a coactivator of pro-inflammatory gene expression in adipocytes and possibly other cell types [11].

In the current study, we investigated the role of NRIP1 in the pathogenesis of psoriasis, including cell proliferation, apoptosis and inflammation. Our research revealed for the first time that NRIP1 mRNA was elevated in the skin lesion, which is in accordance with our previous data of immunohistochemistry showing that expression of NRIP1 was significantly increased both in the epidermis and dermis of PP (data unpublished). Abnormal proliferation of keratinocytes is a important mechanism of psoriasis, resulting in epidermal hyperplasia, a morphological characteristic of psoriasis [12]. Numerous well-established anti-psoriatic treatments, including phototherapy, oral retinoid and topical calcipotriol, could improve the condition of psoriasis patients via influencing keratinocytes proliferation [13]. So, we performed an in vitro study with HaCaT cells to detect the role of NRIP1 in keratinocytes proliferation. Our data showed that suppression of NRIP1 in HaCat cells could significantly inhibit cell growth and induce apoptosis, suggesting that the suppression of cell growth may due to the induction of apoptosis, and indicating that NRIP1 may play a role in cellular proliferation and apoptosis of keratinocytes involved in psoriasis. Our previous study also showed that in breast cancer cells, suppressing NRIP1 could reduce cells proliferation and induce apoptosis [7], however, another study found that overexpression of NRIP1 could reduce the proliferation of colon cancer cells [14]. These contradictory results of NRIP1 indicate that NRIP1 may play diverse roles on cell proliferation in different cell types. So, we need to further confirm our findings on human primary keratinocytes.
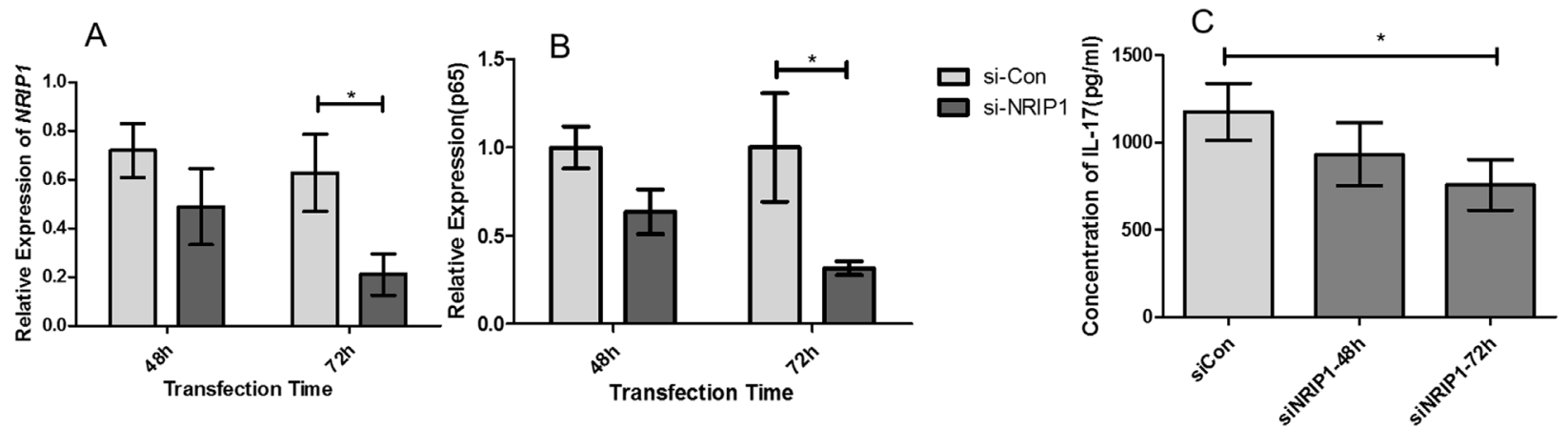

Figure 3: Suppression of NRIP1 reduced expression of $p 65$ and decreased secretion of IL-17 in CD4+T cells. QRT-PCR assay showed that the expression of NRIP1 mRNA A. and $p 65$ mRNA B. in CD4 $4^{+} \mathrm{T}$ cells were reduced significantly at $72 \mathrm{~h}$ after siNRIP1 treatment $(\mathrm{P}<0.05)$. ELISA assay $\mathbf{C}$. showed siNRIP1 transfection decreased the concentration of IL-17 in the supernatants of CD4 ${ }^{+} \mathrm{T}$ cells at $72 \mathrm{~h}$ after siNRIP1 treatment $(\mathrm{p}<0.05)$. 
A

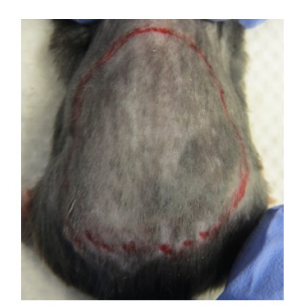

Day 0

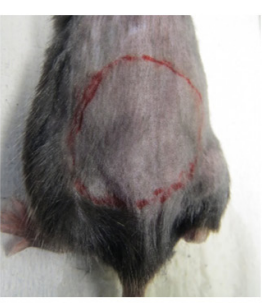

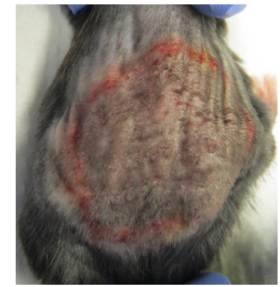

Day 3

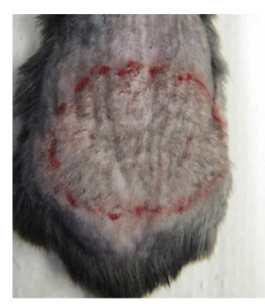

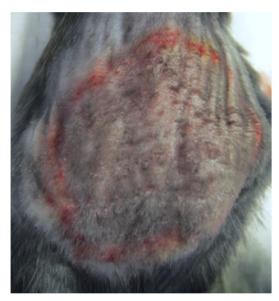

Day 5

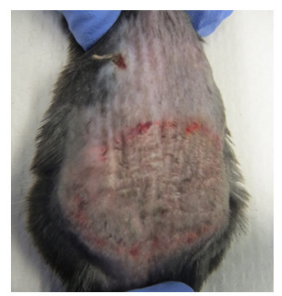

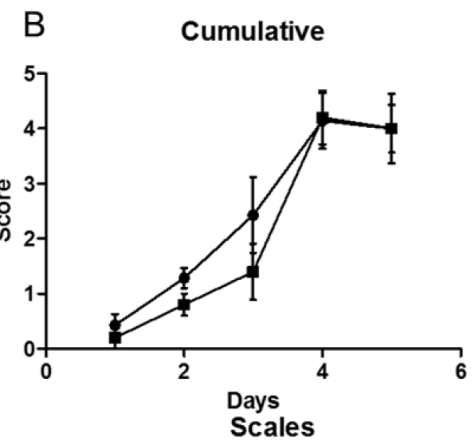

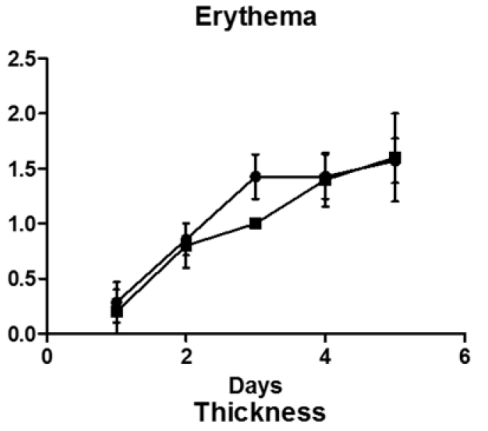

$\rightarrow \mathrm{C} 57 \mathrm{BL} / 6$

$\rightarrow$ Nrip1 KO
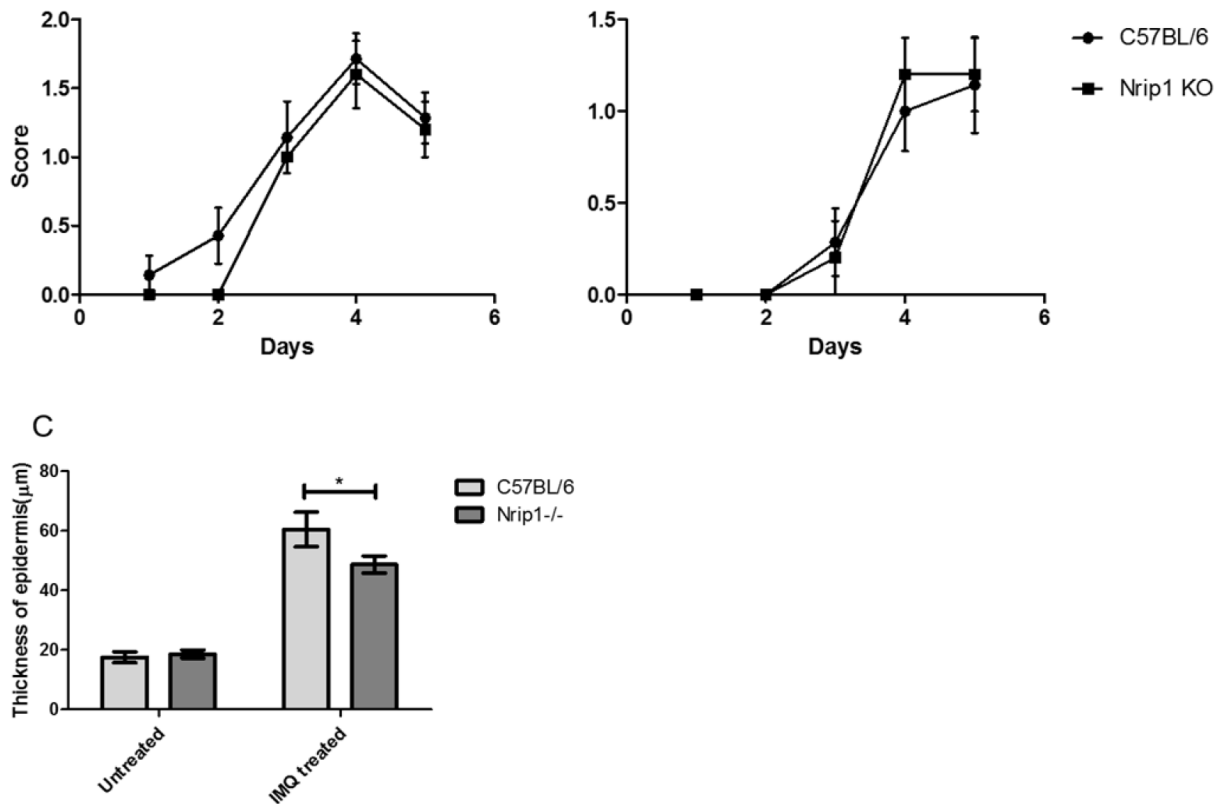

Figure 4: Nrip1 deficiency could delay the IMQ-induced skin inflammation. A. at the third day of IMQ treatment, the skin inflammation of $\mathrm{Nrip}^{-/}$mice was weaker than C57BL/6 WT mice, but at the fifth day, C57BL/6 WT and Nrip $1^{-/}$mice developed similar skin inflammation resembling psoriasis. B. compared with C57BL/6 WT mice, the scores of erythema and scaling of Nrip $1^{-1}$ mice were lower before the third day. From the fourth day, scores of erythema and scaling of both groups are similar. Cumulative scores showed the same trend. There was no difference of thickness scores between the two groups. C. measurement of the thickness of epidermis in H\&E stained skin sections showed that after 5 days of IMQ treatment, thickness of epidermis was increased in both two groups, but the increment was significantly reduced in $\mathrm{Nrip}^{-1 /}$ mice. 
With advances of psoriasis pathogenesis, psoriasis is considered as an autoimmune disease, which is centrally controlled by $\mathrm{T}$ cells [15], interplaying with numerous cell types via different cytokines, such as NF- $\kappa \mathrm{B}[8]$. NF- $\kappa \mathrm{B}$ is a key element regulating inflammation pathways, and it links altered immune cell states and keratinocytes in the pathogenesis of psoriasis [16]. Our data firstly showed that NRIP1 mRNA was increased in PBMCs of psoriasis patients compared to healthy controls. Then we confirmed that the expression of RelA/p65 was elevated in lesions and PBMCs of psoriasis patients, which was in accordance with other previous studies [9]. In order to evaluate the effect of NRIP1 on NF- $\kappa \mathrm{B}$, we detected the expression of p65 in HaCaT cells and primary $\mathrm{CD}^{+} \mathrm{T}$ cells. The results revealed that suppressing NRIP1 in $\mathrm{HaCaT}$ cells and $\mathrm{CD}^{+} \mathrm{T}$ cells could significantly downregulate the expression of RelA/p65. These data suggested that NRIP1 might upregulate the secretion of downstream cytokines by stimulating the expression of RelA/p65 in $\mathrm{CD}^{+} \mathrm{T}$ cells in psoriasis. IL23/Th17 axis was strongly supported to play predominant roles in the pathogenesis of psoriasis in recent studies, studies have showed that the IL-23 pathway is activated and the expression of IL-23 and IL17 were higher in psoriasis patients than healthy controls [17]. The IL-17 level was correlated with the severity of psoriasis [18], and therapies targeting IL-23 and IL-17 had been evaluated with promising effect [19]. In this study, suppression of NRIP1 in CD4 ${ }^{+} \mathrm{T}$ cells also decreased the secretion of IL-17 significantly, suggesting that NRIP1 may involve in psoriasis via upregulating IL-17.

The in vivo data suggested that IMQ-induced skin inflammation was delayed in $\mathrm{Nrip}^{-/}$mice compared to WT mice during the first three days of treatment. The scores of erythema and scaling, and thickness of epidermis of $\mathrm{Nrip}^{-/}$mice were all decreased before the 3rd day. And histological analysis of IMQ-treated skin showed that the density of epidermal ridge and

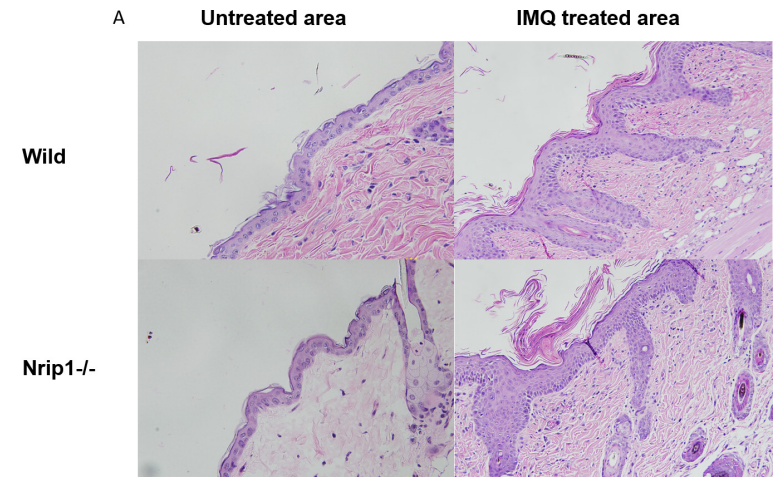

inflammatory cell infiltration in the dermis of $\mathrm{Nrip}^{-/-}$ mice were much less than in WT mice, which suggested that the depletion of Nrip1 may reduce inflammation caused by IMQ treatment. However, on the last day of IMQ treatment, both types of mice had developed similar lesions resembling psoriasis. Thus, we need to further investigate the lesion features on the 3rd day of IMQ treatment, when the lesions of $\mathrm{Nrip}^{-/-}$mice seem to be obviously weaker than those on the WT mice. The IHC results revealed that the expression of p65 in IMQ-treated skin was remarkably reduced in the epidermis and dermis of $\mathrm{Nrip1}^{-/}$mice compared to WT mice, which was in accordance with the data that NRIP1 suppression could downregulate expression of $\mathrm{p} 65$ in patients' $\mathrm{CD}^{+} \mathrm{T}$ cells. These results were attractive, because p65, one important subunit of NF- $\kappa \mathrm{B}$, could suggest the involvement of NF$\kappa \mathrm{B}$ to a certain extent, although we need to further detect the active status of NF- $\kappa \mathrm{B}$ in order to confirm the role of NRIP1 on NF- $\kappa$ B.

As IMQ is a ligand of TLR7 and TLR8 [20], it is also an intense irritant which can induce an obvious inflammatory reaction on skin [21]. The depletion of NRIP1 may only cause a partial decay of pro-inflammatory gene transcription [3]. In other words, NRIP1 may play a role in a subtle state of inflammation, which means NRIP1 deficiency alone is not sufficient to impair the intense inflammatory response to IMQ. This may be the reason that the Nrip1 $1^{-/}$mice developed weaker lesions only during the first three days of IMQ treatment. However, the RelA/p65 expression in the epidermis and dermis of the IMQ treated area was remarkably reduced in $\mathrm{Nrip}^{-/}$mice, suggesting that the depletion of NRIP1 could inhibit the expression of RelA/p65, and also verifying the previous results of human skin, PBMCs, primary keratinocytes and $\mathrm{CD}^{+} \mathrm{T}$ cells.

In conclusion, our studies reveal that NRIP1 might play roles in cellular proliferation and apoptosis of

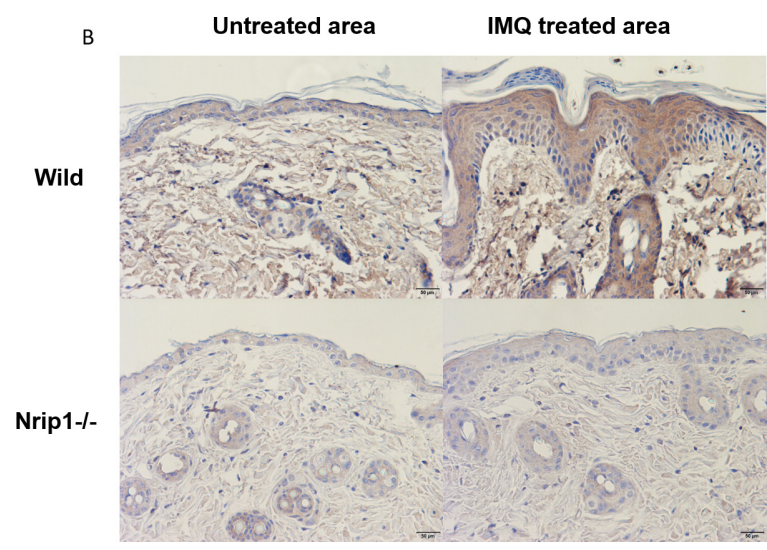

Figure 5: Histology and immunohistochemical (IHC) staining of IMQ treated lesion. A. H\&E (200×), two genotypes of mice showed typical features of psoriasis, including hyper-proliferative keratinocytes, parakeratosis and the absence of granular layer, but the density of epidermal ridge and inflammatory cell infiltration in the dermis of Nrip $1^{-\leftarrow}$ mice were much less than WT mice. B. IHC (200×), p65 positive cells within IMQ treated skin were decreased in both epidermis and dermis of $\mathrm{Nrip}^{-/-}$mice. 
keratinocytes, activation of $\mathrm{CD} 4^{+} \mathrm{T}$ cells and $\mathrm{NF}-\kappa \mathrm{B}$ gene transcription, which are all involved in the pathogenesis of psoriasis, and suggest that NRIP1 may be a novel and attractive therapeutic target for psoriasis. However, more detailed studies are needed to confirm the mechanism behind the function of NRIP1 on inflammation and cell growth and to explore its other potential roles in psoriasis.

\section{MATERIALS AND METHODS}

\section{Skin and blood samples}

Thirty psoriasis patients in progressive status with plaque lesions and 16 healthy volunteers were enrolled for this study. Their characteristics are summarized in Table 1. Two $5 \mathrm{~mm}$ punch biopsies of PP and PN from each patient, as well as one $5 \mathrm{~mm}$ punch of NN, were obtained. Five $\mathrm{ml}$ peripheral blood was also obtained from each donor. The diagnosis of psoriatic patients was performed by a consultant dermatologist, who also provided patient demographic information and disease parameters along with psoriasis area and severity index (PASI). Healthy controls were defined as people who had no history of psoriasis and no skin lesions on clinical examination. Each member of the healthy control group had a qualified match in age and sex with the psoriatic patient group. All samples were collected in accordance with the ethical guidelines mandated by the ethical committee of the Institute of Dermatology, Chinese Academy of Medical Sciences. Informed consent was signed by those who agreed to participate in this study. All skin biopsy specimens were immediately stored in liquid nitrogen for real time quantitative polymerase chain reaction (qRT-PCR) experiments.

\section{HaCaT cells}

HaCaT cells were cultured in Dulbecco's modified Eagle's medium (DMEM) supplemented with 10\% fetal bovine serum (FBS), $100 \mathrm{U} / \mathrm{ml}$ penicillin, and $100 \mu \mathrm{g} /$ $\mathrm{ml}$ streptomycin, and incubated at $37^{\circ} \mathrm{C}$ in a humidified atmosphere containing 5\% $\mathrm{CO} 2$.

\section{NRIP1 shRNA transfection}

$\mathrm{HaCaT}$ cells were seeded in a six-well plate with $60 \%$ of confluence in DMEM with $10 \% \mathrm{FBS}$ and polybrene (8 $\mu \mathrm{g} / \mathrm{ml})$. Lentiviral particles $(20 \mu \mathrm{L} / \mathrm{mL})$ containing shRNA of human NRIP1 (Santa Cruz, CA) were added to the cells and incubated for $24 \mathrm{~h}$, then medium was replaced with fresh growth medium, cells were cultured for another 24 h. Puromycin $(0.25 \mu \mathrm{g} / \mathrm{mL}$; Sigma-Aldrich) was used to select the stable clones expressing target shRNA. The culture medium was changed every 2-3 days, until resistant colonies could be identified. Non sense shRNA (shCON) lentiviral particles (Santa Cruz, CA) were added to the control cells. The expression of NRIP1 in stable cells was detected by qRT-PCR.

\section{MTT assay}

To evaluate the viability of shRNA treated HaCaT cells, at 24, $48,72,96$ and $120 \mathrm{~h}$ after being seeded in 6 -well plates $\left(5 \times 10^{5}\right.$ cells/well $), 100 \mu \mathrm{l}$ of $5 \mathrm{mg} / \mathrm{ml}$ 3-(4,5-dimethylthiazol-2-yl)-2,5-diphenyltetrazolium bromide (MTT, Sigma, St. Louis, MO) and $1.9 \mathrm{ml}$ serumfree culture medium were added to each well. Following a four-hour incubation, wells were aspirated and $2 \mathrm{~mL}$ of DMSO was added to each MTT-treated well. Each well was divided into 10 wells of a 96-well-plate, and the absorption at $540 \mathrm{~nm}$ was measured by spectrophotometry (BioTek PowerWave XS, Winooski, VT, US). An MTT assay for each cell line was repeated 3 times.

\section{Propidium iodide (PI) staining}

For cell cycle and apoptosis analysis, $\mathrm{HaCaT}$ cells were transfected with shRNA as described above, then were trypsinized and resuspended in PBS with $0.1 \%$ bovine serum albumin. A total of $1 \times 10^{6}$ cells were fixed in $25 \%$ ethanol overnight at $4^{\circ} \mathrm{C}$. The cells were then stained with PI ( $50 \mathrm{mg} / \mathrm{mL})$ containing RNase A $(0.7 \mathrm{mg} / \mathrm{mL})$, and incubated at $37^{\circ} \mathrm{C}$. 10,000 events were collected for each sample, and examined by flow cytometry (BD Accuri C6, Franklin Lakes, NJ). Each group was assayed three times.

\section{Annexin-V staining}

shRNA induced apoptosis in $\mathrm{HaCaT}$ cells was verified and evaluated by Annexin-V staining. The cells were stained with PE Annexin-V and 7-AminoActinomycin (7-AAD) following the manufacturer's instructions (BD Pharmingen, Franklin Lakes, NJ) to detect early apoptosis cells (PE Annexin- $\mathrm{V}^{+} / 7-\mathrm{AAD}^{-}$ events) and late apoptosis cells (PE Annexin- $\mathrm{V}^{+} / 7$ $\mathrm{AAD}^{+}$events) and examined by flow cytometry (BD Accuri C6). Apoptosis of each group was assayed three times.

\section{Western blot analysis}

About $1 \times 10^{6} \mathrm{HaCaT}$ cells were lysed in RIPA Lysis Buffer for $30 \mathrm{~min}$, then collected and measured by using the BCA Protein Assay Reagent Kit (Pierce, Biotechnology). Equal amounts of protein from each sample were mixed with $1 \times$ loading buffer and denatured at $95{ }^{\circ} \mathrm{C}$ for $5 \mathrm{~min}$, then they were subjected to $10 \% \mathrm{SDS}$ PAGE and transferred on to polyvinylidene difluoride membranes (Roche, Germany). After being blocked for $2 \mathrm{~h}$ with $5 \%$ bovine serum albumin in $0.1 \%$ Tween-20/ TBS, the PVDF membranes were incubated with primary antibody against Human p65 (Abcam, UK) at a concentration of $1: 1000$ at $4^{\circ} \mathrm{C}$ overnight. After being washed, the membranes were incubated with HRPconjugated secondary antibody at 1: 3000 dilutions at room temperature for $2 \mathrm{~h}$. After being washed again, protein bands were detected by enhanced chemiluminescence. The 
Table 1: Demographics and disease parameters of patients with psoriasis

\begin{tabular}{|c|c|c|c|c|c|c|}
\hline Case & Sex & Age (years) & Nail involved & Family history & $\begin{array}{c}\text { Lengh of } \\
\text { disease (years) }\end{array}$ & PASI score \\
\hline 1 & $\mathrm{~F}$ & 45 & & & 3 & 46.2 \\
\hline 2 & $\mathrm{M}$ & 33 & & + & 5 & 35.4 \\
\hline 3 & $\mathrm{~F}$ & 50 & & & 10 & 5.8 \\
\hline 4 & $\mathrm{M}$ & 41 & + & + & 10 & 16.2 \\
\hline 5 & $\mathrm{~F}$ & 22 & & & 1 & 8.4 \\
\hline 6 & $\mathrm{M}$ & 33 & & & 3 & 36.3 \\
\hline 7 & $\mathrm{M}$ & 35 & + & & 11 & 9.4 \\
\hline 8 & $\mathrm{M}$ & 34 & + & & 6 & 11.6 \\
\hline 9 & $\mathrm{M}$ & 26 & & & 1 & 20.2 \\
\hline 10 & $\mathrm{M}$ & 52 & & & 2 & 26.3 \\
\hline 11 & $\mathrm{M}$ & 32 & & + & 1 & 15.2 \\
\hline 12 & $\mathrm{M}$ & 23 & & & 8 & 14.8 \\
\hline 13 & $\mathrm{M}$ & 44 & & & 7 & 5.2 \\
\hline 14 & $\mathrm{M}$ & 50 & + & + & 10 & 9.2 \\
\hline 15 & M & 20 & & & 8 & 5.4 \\
\hline 16 & $\mathrm{M}$ & 51 & + & + & 6 & 16.3 \\
\hline 17 & $\mathrm{M}$ & 59 & & & 10 & 26.4 \\
\hline 18 & $\mathrm{M}$ & 48 & & & 5 & 15.5 \\
\hline 19 & $\mathrm{M}$ & 48 & & & 8 & 22.6 \\
\hline 20 & $\mathrm{M}$ & 51 & & & 8 & 25.5 \\
\hline 21 & $\mathrm{M}$ & 25 & & & 1 & 12.4 \\
\hline 22 & $\mathrm{M}$ & 48 & & & 20 & 8.5 \\
\hline 23 & $\mathrm{M}$ & 25 & + & & 1 & 15.5 \\
\hline 24 & $\mathrm{M}$ & 25 & + & & 1 & 26.3 \\
\hline 25 & $\mathrm{~F}$ & 48 & & & 3 & 8.6 \\
\hline 26 & $\mathrm{~F}$ & 28 & & & 1 & 14.4 \\
\hline 27 & $\mathrm{~F}$ & 25 & & + & 10 & 4.3 \\
\hline 28 & $\mathrm{~F}$ & 22 & & & 2 & 16.2 \\
\hline 29 & $\mathrm{~F}$ & 51 & & + & 19 & 20.5 \\
\hline 30 & $\mathrm{~F}$ & 47 & & & 3 & 8.6 \\
\hline
\end{tabular}

images were acquired by FluorChem FC2 System (Alpha Innotech Corporation, USA). The membrane was stripped and reprobed with GAPDH antibody.

\section{$\mathrm{CD4}^{+} \mathrm{T}$ cells}

Firstly, PBMCs were isolated from peripheral blood of psoriasis patients by Ficoll-Paque density gradient centrifugation, and then $\mathrm{CD}^{+} \mathrm{T}$ cells were isolated using BD IMag TM human $\mathrm{CD}^{+}{ }^{+} \mathrm{T}$ cells enrichment set according to manufacturer's protocol (BD, USA). Briefly, for every $10^{7}$ cells, $50 \mu \mathrm{BD}$ IMag ${ }^{\mathrm{TM}} \mathrm{CD}^{+}$beads were added, mixed thoroughly, and incubated at room temperature for 30 mins. Following that, $1 \mathrm{ml}$ BD IMag TM buffer was added, and cells were transferred into FACS tubes and placed in strong magnetic field for 8-10 min. $\mathrm{CD} 4^{+} \mathrm{T}$ cells were then attached onto the internal wall of the tubes. The supernatants were carefully removed, and then the tubes were placed out of the magnetic field and washed twice by using the IMag TM buffer. Finally, the supernatants were discarded and the remaining cells were collected for the following experiments.

\section{NRIP1 siRNA transfection}

The RNA interference (siRNA) sequence for silencing human NRIPI was purchased from Genepharma (Shanghai, China). The sequences are as follows: 
siNRIP1: FP-5'GAGGAUCAGAACUUUAACATT3', RP-5'UGUUAAAGUUCUGACCUCTT3'. Negative control of siRNA ( control. Transfection of the siRNAs were performed using Lipofectamine ${ }^{\circledR} 2000$ (Thermo Fisher Scientific, Waltham, MA, USA) according to the manufacturer's protocol. $\mathrm{CD}^{+} \mathrm{T}$ cells were seeded at a density of $3 \times 10^{5}$ cells/well in 6-well plates, when the cells reached 60$80 \%$ confluency, the siRNAs- Lipofectamine ${ }^{\circledR} 2000$ complexes were prepared and added to each well. After $6 \mathrm{~h}$ of incubation, the medium was replaced with fresh culture medium. The medium was replaced with $2 \mathrm{ml}$ fresh pre-warmed medium per well every $24 \mathrm{~h}$. After $48 \mathrm{~h}$ of siRNA transfection, the cells were harvested for qRT-PCR.

\section{qRT-PCR}

Total RNA was extracted from $2 \times 10^{6}$ cells using TRIZOL (Invitrogen/Thermo Fisher Scientific, Waltham, MA, USA) according to the manufacturer's protocol, and the quantity of RNA was determined by a spectrophotometer at $260 \mathrm{~nm} .20 \mu \mathrm{l}$ of cDNA was synthesized from $1 \mu \mathrm{g}$ of the total RNA using RevertAid ${ }^{\mathrm{TM}}$ First Strand cDNA Synthesis Kit (MBI Fermentas, Ontario, Canada). qRT-PCR analysis was performed with SYBR $^{\circledR}$ Premix Ex Taq ${ }^{\mathrm{TM}}$ (TaKaRa Co., Dalian, China). Following the manufacturer's protocol, $1 \mu \mathrm{L}$ RT cDNA was mixed with $0.6 \mu \mathrm{L}$ forward, $0.6 \mu \mathrm{L}$ reverse primers $(10 \mu \mathrm{M}), 10 \mu \mathrm{L}$ Premix Ex TaqTM, and $8.4 \mu \mathrm{L}$ Nucleasefree water to obtain a final volume of $20 \mu \mathrm{L}$. qRT-PCR reactions were run on an Applied Biosystems 7300 RealTime PCR System (Applied Biosystems, Foster City, CA, USA). The amplification conditions were $95{ }^{\circ} \mathrm{C}$ for 10 min, and 40 cycles of $95^{\circ} \mathrm{C}$ for $30 \mathrm{~s}, 60^{\circ} \mathrm{C}$ for $30 \mathrm{~s}$ and $72{ }^{\circ} \mathrm{C}$ for $30 \mathrm{~s}$. The levels for target gene mRNA were normalized to GAPDH in the same sample. For NRIP1 primer, forward, 5'- GCTGGGCATAATGAAGAGGA-3', reverse, 5'- CAAAGAGGCCAGTAATGTGCTATC-3'. For RelA/p65 primer, forward, 5'-CTGCAGTTTGA TGATGAAGA-3', reverse, 5'-TAGGCGAGTTATAGCC TCAG-3'. For GAPDH primer, forward, 5'- ATGGG GAAGGTGAAGGTCG-3', and reverse, 5'- GGGGT CATTGA TGGCAACAATA-3'. qRT-PCR assays for each sample were repeated 3 times.

\section{Enzyme-linked immuno sorbent assay (ELISA)}

CD4 ${ }^{+} \mathrm{T}$ cells were transfected with RNA interference (siRNA) sequences and supernatants were collected at $48 \mathrm{~h}$ and $72 \mathrm{~h}$ after transfection. The concentration of IL-17 was measured by using ELISA kits (ELH-IL1, 7RayBiotech, GA, USA). One-hundred $\mu$ of each standard and supernatant sample were added into the 96-well plate coated with anti-Human IL17 and incubated over night at $4^{\circ} \mathrm{C}$ with gentle shaking, and washed 4 times with washing buffer. Wells were incubated for 1 hour with IL-17 specific biotinylated antibody, rinsed 4 times with wash buffer, reacted with streptavidin solution diluent for 45 minutes, washed 4 times with wash buffer, and then incubated for 30 minutes with $100 \mu \mathrm{l}$ TMB One-Step substrate reagent in the dark. The plates were quenched with stop solution and the absorption at $450 \mathrm{~nm}$ was measured by spectrophotometry (BioTek PowerWave XS, Winooski, VT, US). ELISA assays for each sample were repeated 3 times.

\section{Mice and treatments}

10- to 12-month-old C57BL/6J (B6) and Nrip1 $1^{-1-}$ mice were used. The Nripl knockout mice had been backcrossed to B6 for more than 10 generations before the homozygous knockout (Nrip $\mathrm{I}^{-1}$ ) mice were generated. The animal housing conditions are described previously [7]. Animal care and handling were conducted according to NIH guidelines and the policies of Southern Illinois University School of Medicine Laboratory Animal Care and Use Committee, Springfield, IL. Both groups of mice received a daily topical dose of $62.5 \mathrm{mg}$ IMQ cream (5\%) (Aldara; 3M Pharmaceuticals) on their shaved backs for 5 consecutive days.

\section{Scoring severity of skin inflammation}

We scored the severity of inflammation of the back skin based on the clinical Psoriasis Area and Severity Index (PASI) [20]. And we scored erythema, scaling, and thickening independently on a scale from 0 to 4 , and the cumulative score from 0 to 12 .

\section{Histology and IHC assay}

Samples from back skin (3 $\mathrm{mm}$ diameter) were excised and fixed overnight in 10\% neutral buffered formalin, then transferred to PBS (pH 7.4), and embedded in paraffin. Sections ( $4 \mu \mathrm{m}$ thick) of each specimen were cut for H\&E staining and IHC study.

Tissue slides were deparaffinized with xylene and then rehydrated with decreasing ethanol concentrations. Antigen retrieval was conducted by boiling-bath method in $0.01 \mathrm{M}$ sodium citrate buffer, $\mathrm{pH} 6.0$ to about $95^{\circ} \mathrm{C}$, and then slides were put in the buffer for $15 \mathrm{~min}$. Blocking solution was used to prevent nonspecific binding of antibodies. Sections were incubated with polyclonal antiNRIP1 antibody (SCBT, Santa Cruz, CA, 1:50 dilution) at $4^{\circ} \mathrm{C}$ overnight. HRP Detection System (Dako EnVision System HRP; Dako North America, Inc. Carpinteria, CA), was used for detection. After counterstaining with hematoxylin (Harris Modified Hematoxylin, Fisher Scientific, Fairlawn, New Jersey), the sections were dehydrated and mounted. The specific staining of NRIP1 in the sections was examined microscopically (Olympus, Center Valley, PA, USA). 


\section{Statistical analysis}

All data were analyzed with Prism 5.0 (GraphPad Software Inc., San Diego, CA, USA) and expressed as means \pm SE. T-test and one-way analysis of variance (ANOVA) were used for data analysis. Differences with $\mathrm{P}$ $<0.05$ were considered significant.

\section{ACKNOWLEDGMENTS}

The authors would like to thank Dr Fang Fang, Dr Xinfeng Wu, Dr Yan Wang, Dr Liming Huang, Chunyan Cao, Xiaoxia Guo, Yan Yan and Xiaoli Fan for their assistance in collecting materials, and all study subjects for providing samples of skin and blood. Lisa Hensley and Melissa Dodds helped edit the manuscript. This work was supported by the grants from NIH grants (R03 AG046605 and K01 AG046432).

\section{CONFLICTS OF INTEREST}

The authors state no conflicts of interest.

\section{REFERENCES}

1. Christian M, Kiskinis E, Debevec D, Leonardsson G, White R, Parker MG. RIP140-targeted repression of gene expression in adipocytes. Mol Cell Biol. 2005; 25:9383-9391.

2. Herzog B, Hallberg M, Seth A, Woods A, White R, Parker MG. The nuclear receptor cofactor, receptor-interacting protein 140 , is required for the regulation of hepatic lipid and glucose metabolism by liver $\mathrm{X}$ receptor. Mol Endocrinol. 2007; 21:2687-2697.

3. Augereau P, Badia E, Carascossa S, Castet A, Fritsch S, Harmand PO, Jalaguier S, Cavailles V. The nuclear receptor transcriptional coregulator RIP140. Nucl Recept Signal. 2006; 4:e24.

4. Zschiedrich I, Hardeland U, Krones-Herzig A, Berriel DM, Vegiopoulos A, Muggenburg J, Sombroek D, Hofmann TG, Zawatzky R, Yu X, Gretz N, Christian M, White R, Parker MG, Herzig S. Coactivator function of RIP140 for NFkappaB/RelA-dependent cytokine gene expression. Blood. 2008; 112:264-276.

5. Nautiyal J, Steel JH, Rosell MM, Nikolopoulou E, Lee K, Demayo FJ, White R, Richards JS, Parker MG. The nuclear receptor cofactor receptor-interacting protein 140 is a positive regulator of amphiregulin expression and cumulus cell-oocyte complex expansion in the mouse ovary. Endocrinology. 2010; 151:2923-2932.

6. Docquier A, Harmand PO, Fritsch S, Chanrion M, Darbon JM, Cavailles V. The transcriptional coregulator RIP140 represses E2F1 activity and discriminates breast cancer subtypes. Clin Cancer Res. 2010; 16:2959-2970.

7. Aziz MH, Chen X, Zhang Q, DeFrain C, Osland J, Luo Y, Shi X, Yuan R. Suppressing NRIP1 inhibits growth of breast cancer cells in vitro and in vivo. Oncotarget. 2015; 6:39714-39724. doi: 10.18632/oncotarget.5356.

8. Goldminz AM, Au SC, Kim N, Gottlieb AB, Lizzul PF. NF-kappaB: an essential transcription factor in psoriasis. $\mathrm{J}$ Dermatol Sci. 2013; 69:89-94.

9. Lizzul PF, Aphale A, Malaviya R, Sun Y, Masud S, Dombrovskiy V, Gottlieb AB. Differential expression of phosphorylated NF-kappaB/RelA in normal and psoriatic epidermis and downregulation of NF-kappaB in response to treatment with etanercept. J Invest Dermatol. 2005; 124:1275-1283.

10. Tsuruta D. NF-kappaB links keratinocytes and lymphocytes in the pathogenesis of psoriasis. Recent Pat Inflamm Allergy Drug Discov. 2009; 3:40-48.

11. Takata A, Otsuka M, Kojima K, Yoshikawa T, Kishikawa T, Yoshida H, Koike K. MicroRNA-22 and microRNA-140 suppress NF-kappaB activity by regulating the expression of NF-kappaB coactivators. Biochem Biophys Res Commun. 2011; 411:826-831.

12. Boehncke WH, Schon MP. Psoriasis. Lancet. 2015; 386:983-994.

13. Raut AS, Prabhu RH, Patravale VB. Psoriasis clinical implications and treatment: a review. Crit Rev Ther Drug Carrier Syst. 2013; 30:183-216.

14. Lapierre M, Bonnet S, Bascoul-Mollevi C, Ait-Arsa I, Jalaguier S, Del RM, Plateroti M, Roepman P, Ychou M, Pannequin J, Hollande F, Parker M, Cavailles V. RIP140 increases APC expression and controls intestinal homeostasis and tumorigenesis. J Clin Invest. 2014; 124:1899-1913.

15. Eberle FC, Bruck J, Holstein J, Hirahara K, Ghoreschi K. Recent advances in understanding psoriasis. F1000Res. 2016; 5.

16. Tsuruta D. NF-kappaB links keratinocytes and lymphocytes in the pathogenesis of psoriasis. Recent Pat Inflamm Allergy Drug Discov. 2009; 3:40-48.

17. Fitch E, Harper E, Skorcheva I, Kurtz SE, Blauvelt A. Pathophysiology of psoriasis: recent advances on IL-23 and Th17 cytokines. Curr Rheumatol Rep. 2007; 9:461-467.

18. Grine L, Dejager L, Libert C, Vandenbroucke RE. An inflammatory triangle in psoriasis: TNF, type I IFNs and IL-17. Cytokine Growth Factor Rev. 2015; 26:25-33.

19. Marinoni B, Ceribelli A, Massarotti MS, Selmi C. The Th17 axis in psoriatic disease: pathogenetic and therapeutic implications. Auto Immun Highlights. 2014; 5:9-19.

20. van der Fits L, Mourits S, Voerman JS, Kant M, Boon L, Laman JD, Cornelissen F, Mus AM, Florencia E, Prens EP, Lubberts E. Imiquimod-induced psoriasis-like skin inflammation in mice is mediated via the IL-23/IL-17 axis. J Immunol. 2009; 182:5836-5845.

21. Bai S, Zhang Z, Hou S, Liu X. Influence of different types of contact hypersensitivity on imiquimod-induced psoriasis-like inflammation in mice. Mol Med Rep. 2016; 14:671-680. 